\title{
CONTINUITY PROPERTIES OF THE SPECTRUM OF OPERATORS ON LEBESGUE SPACES
}

\author{
BRUCE A. BARNES \\ (Communicated by John B. Conway)
}

\begin{abstract}
Fix $1 \leq p<s \leq \infty$. Let $T_{x}, x \in[p, s]$, be the collection of bounded linear operators on the Lebesgue spaces $L^{x}$ determined by some fixed operator $T$. This paper concerns continuity properties of the map $x \rightarrow \sigma\left(T_{x}\right)$.
\end{abstract}

\section{INTRODUCTION}

Let $\Omega$ be a nonempty set, and let $\mu$ be a $\sigma$-finite measure on $\Omega$. If $1 \leq$ $p \leq \infty$, then let $L^{p}$ denote the usual Lebesgue space $L^{p}(\Omega, \mu)$ with the usual $p$-norm. Now fix $p$ and $s$ with $1 \leq p<s \leq \infty$. Let $B_{p, s}$ be the algebra of all linear operators $T: L^{p} \cap L^{s} \rightarrow L^{p} \cap L^{s}$ with the property that $T$ is continuous on $L^{p} \cap L^{s}$ with respect to both the $p$-norm and the $s$-norm. The algebra $\mathscr{B}_{p, s}$ is a Banach algebra which is studied in [2]. Also, some applications involving $\mathscr{B}_{p, s}$ can be found in [3]. If $T \in \mathscr{B}_{p, s}$, then by the Riesz Convexity Theorem $[6$, Theorem 11, p. 525] for each $x$ in the interval $[p, s]$ the linear operator $T$ has a unique extension to a bounded linear operator $T_{x}$ on $L^{x}$ (in the case where $s=\infty, T_{\infty}$ is a bounded linear operator on the closure of $L^{p} \cap L^{\infty}$ in $\left.L^{\infty}\right)$. Let $\sigma\left(T_{x}\right)$ and $r\left(T_{x}\right)$ denote the spectrum and spectral radius of this extension. It is well known that $\sigma\left(T_{x}\right)$ can be different for different $x$ in $[p, s]$. An example of this phenomenon is given in [4]: Let $\Omega=(0, \infty)$ and $\mu$ be the Lebesgue measure. Take $T$ to be the Cesàro operator, so

$$
T_{y}(f)(t)=t^{-1} \int_{0}^{t} f(x) d x \quad\left(f \in L^{y}\right),
$$

$1<y \leq \infty$. Then $\sigma\left(T_{y}\right)$ is the circle with center and radius $\left(2\left(1-y^{-1}\right)\right)^{-1}$. Note that $\sigma\left(T_{y}\right)$ varies continuously in $y$ in this case.

In this paper we study continuity properties of the map $y \rightarrow \sigma\left(T_{y}\right)$. A key theorem here is that for $T \in \mathscr{B}_{p, s}$, the function $y \rightarrow r\left(T_{y}\right)$ is continuous on the open interval $(p, s)$ (it need not be continuous on $[p, s]$ ). A number of other continuity results are derived using this basic theorem as a tool.

Received by the editors July $26,1988$.

1980 Mathematics Subject Classification (1985 Revision). Primary 47A10.

Key words and phrases. Linear operator, spectrum, spectral radius. 
There are only a few examples in the literature where $\sigma\left(T_{y}\right)$ is specifically computed and this set is different for different values of $y$. Specific examples where this occurs can be found in [4], [5], and [7]. Next we give a number of additional examples.

Example 1. Let $\Omega$ be the positive integers, and let $\mu$ be the measure with

$$
\mu(k)=1 / k ! \quad(k \geq 1) .
$$

For $1 \leq p<\infty$, let $T_{p}$ be the linear operator on $l^{p}(\mu)$ defined by $T_{p}\left(\left\{a_{k}\right\}\right)=$ $\left\{b_{k}\right\}$ where $b_{k}=(k+1)^{-1} a_{k+1}, k \geq 1$. Also, let $T_{\infty}$ act on $c_{0}$ by the same rule.

Note. For $1<p \leq \infty, T_{p}$ is compact and $\sigma\left(T_{p}\right)=\{0\}$.

Proof. Fix $p, 1<p<\infty$. Define $M_{n}$ on $l^{p}(\mu)$ by $M_{n}\left(\left\{a_{k}\right\}\right)=\left\{b_{k}\right\}$ where $b_{k}=a_{k}$ for $1 \leq k \leq n$, and $b_{k}=0$ for $k>n$. For $\left\{a_{k}\right\} \in l^{p}(\mu)$,

$$
\begin{aligned}
\left\|\left(T_{p}-M_{n} T_{p}\right)\left\{a_{k}\right\}\right\|_{p}^{p} & =\sum_{k=n+1}^{\infty}(1 / k !)(k+1)^{-p}\left|a_{k+1}\right|^{p} \\
& =\sum_{k=n+1}^{\infty}(k+1)^{1-p}(1 /(k+1) !)\left|a_{k+1}\right|^{p} \leq(n+2)^{1-p}\left\|\left\{a_{k}\right\}\right\|_{p}^{p} .
\end{aligned}
$$

Therefore

$$
\left\|T_{p}-M_{n} T_{p}\right\|_{p} \leq(n+2)^{p^{-1}-1} \rightarrow 0
$$

as $n \rightarrow \infty$. This proves $T_{p}$ is compact. A similar computation shows that $T_{\infty}$ is compact.

Now it is easy to see that the operator $M_{n} T_{p}$ is nilpotent, so $\sigma\left(M_{n} T_{p}\right)=\{0\}$ for $n \geq 1$. Since $T_{p}$ is compact, this implies $\sigma\left(T_{p}\right)=\{0\}$ [8, Theorem 3].

Now we consider the case where $p=1$. Let $l^{1}$ denote the usual sequence space (the $L^{1}$-space of the positive integers with respect to counting measure). Define an isometry $W_{1}: l^{1} \rightarrow l^{1}(\mu)$ by $W_{1}\left(\left\{a_{k}\right\}\right)=\left\{c_{k}\right\}$ where $c_{k}=k ! a_{k}$, $k \geq 1$. The isometry $W_{1}$ maps $l^{1}$ onto $l^{1}(\mu)$. Let $B$ be the unilateral backward shift on $l^{1} ; B\left(\left\{a_{k}\right\}\right)=\left\{b_{k}\right\}$ where $b_{k}=a_{k+1}, k \geq 1$. A straightforward computation shows

$$
W_{1}^{-1} T_{1} W_{1}=B \quad \text { on } l^{1} .
$$

As is well known, $\sigma(B)=D$, the closed unit disk, so $\sigma\left(T_{1}\right)=D$.

To summarize: in this case $T \in \mathscr{B}_{1, \infty}, T_{x}$ is compact with $\sigma\left(T_{x}\right)=\{0\}$ for $1<x \leq \infty$, while $T_{1}$ is not compact with $\sigma\left(T_{1}\right)=D$. The spectral radius function is given by

$$
r\left(T_{x}\right)=0 \quad \text { for } 1<x \leq \infty, \quad r\left(T_{1}\right)=1 .
$$

Example II. Let $\Omega$ be the positive integers, and let $\mu$ be the measure with

$$
\mu(k)=2^{k} \quad(k \geq 1) .
$$


Let $S$ and $B$ be defined on any sequence space by

$$
\begin{array}{ll}
S\left(\left\{a_{k}\right\}\right)=\left\{b_{k}\right\} & \text { where } b_{1}=0, b_{k}=a_{k-1} \text { for } k>1 ; \\
B\left(\left\{a_{k}\right\}\right)=\left\{c_{k}\right\} & \text { where } c_{k}=a_{k+1} \text { for } k \geq 1 .
\end{array}
$$

Let $S_{p}$ and $B_{p}$ denote the operators $S$ and $B$ acting on the sequence space $l^{p}(\mu), 1 \leq p<\infty$. Let $l^{p}$ be the usual $L^{p}$-space on the positive integers with counting measure. For $1 \leq p<\infty$ define $W_{p}: l^{p} \rightarrow l^{p}(\mu)$ by $W_{p}\left(\left\{a_{k}\right\}\right)=\left\{d_{k}\right\}$ where $d_{k}=2^{-k / p} a_{k}, k \geq 1$. Then $W_{p}$ is a linear isometry of $l^{p}$ onto $l^{p}(\mu)$. Each of the following properties is established by a simple computation.

(II.1) $S_{2}^{*}=2 B_{2}$, so $S_{2}+2 B_{2}$ is selfadjoint on $l^{2}(\mu)$;

(II.2) $W_{p}^{-1} S_{p} W_{p}=2^{1 / p} S$ on $l^{p}$; $W_{p}^{-1} B_{p} W_{p}=2^{-1 / p} B$ on $l^{p}$.

Now the spectrum of an operator of the form $a+b S+c B, a, b, c \in \mathbf{C}$, on the space $l^{p}$ has been specifically computed by I. Gokhberg and M. Zambitsky. This result can be found in [9, Proposition 2]. Combining this information with (II.1)-(II.3), we can compute the spectrum of any operator of the form $a+b S_{p}+c B_{p}, 1 \leq p<\infty$. We state these results for a specific operator:

Set $T_{p}=S_{p}+2 B_{p} \in \mathscr{B}\left(l^{p}(\mu)\right), 1 \leq p<\infty$. Then

(II.4) $\sigma\left(T_{2}\right)=\{\lambda \in \mathbf{R}:-2 \sqrt{2} \leq \lambda \leq 2 \sqrt{2}\}$;

(II.5) For $p \neq 2, p^{-1}+q^{-1}=1\left(q^{-1}=0\right.$ when $\left.p=1\right), \sigma\left(T_{p}\right)$ is the set of all $\lambda=x+i y, x, y \in \mathbf{R}$, which lie inside or on the ellipse $x^{2} / a^{2}+y^{2} / b^{2}=1$ where $a=\left(2^{1 / p}+2^{1 / q}\right)$ and $b=\left(2^{1 / p}-2^{1 / q}\right)$.

(II.6) $r\left(T_{p}\right)=2^{1 / p}+2^{1-1 / p}, 1 \leq p<\infty$.

The operator $T_{2}$ in this example is selfadjoint. In this case it is always true that when $2 \leq x \leq y$ or $y \leq x \leq 2$, then $\sigma\left(T_{x}\right) \subseteq \sigma\left(T_{y}\right)$; see [2, Theorem 4.4]. Also, the fact that $\sigma\left(T_{p}\right) \neq \sigma\left(T_{2}\right)$ for $p \neq 2$ shows that the hypothesis (4.1) in $\S 4$ of [1] cannot be omitted and still obtain the results in [1, Theorem 4.8].

\section{CONTINUITY PROPERTIES}

The main result of this paper concerns the continuity of the spectral radius function $x \rightarrow r\left(T_{x}\right)$; this result is stated in Theorem 3. On the way to proving this theorem, we derive some additional interesting continuity properties of the spectrum.

Proposition 1. Assume $T \in \mathscr{B}_{p, s}$ and $r\left(T_{y}\right)=0$ for some $y \in[p, s]$. Then $r\left(T_{x}\right)=0$ for all $x \in(p, s)$.

Proof. Fix $x \in(p, s)$. We may assume $x<y$. Choose $z$ such that $z \in(p, s)$ and $z<x$. Then $\exists t \in(0,1)$ with $x^{-1}=t z^{-1}+(1-t) y^{-1}$. Applying the Riesz Convexity Theorem, we have for all $n \geq 1$

$$
\left\|\left(T^{n}\right)_{x}\right\| \leq\left\|\left(T^{n}\right)_{z}\right\|^{t}\left\|\left(T^{n}\right)_{y}\right\|^{1-t} .
$$


Therefore

$$
r\left(T_{x}\right) \leq r\left(T_{z}\right)^{t} r\left(T_{y}\right)^{1-t}=0 .
$$

Corollary 2. Assume $T \in \mathscr{B}_{p, s}$ and for some $y \in[p, s] \sigma\left(T_{y}\right)$ is finite. Then $\sigma\left(T_{x}\right)=\sigma\left(T_{y}\right)$ for all $x \in(p, s)$.

Proof. Assume $\sigma\left(T_{y}\right)=\left\{\lambda_{1}, \ldots, \lambda_{n}\right\}$. Let $q$ be the polynomial $q(z)=$ $\prod_{k=1}^{n}\left(z-\lambda_{k}\right)$. Then $\sigma\left(q\left(T_{y}\right)\right)=q\left(\sigma\left(T_{y}\right)\right)=\{0\}$. It follows from Proposition 1 that $\sigma\left(q\left(T_{x}\right)\right)=\{0\}$ for all $x \in(p, s)$. Thus, $\sigma\left(T_{x}\right) \subseteq\left\{\lambda_{1}, \ldots, \lambda_{n}\right\}$ for all such $x$. Fix any $x \in(p, s), x \neq y$. We may assume $x<y$. Now $T \in \mathscr{B}_{x, y}$, and both $\sigma\left(T_{x}\right)$ and $\sigma\left(T_{y}\right)$ are finite. Then by [2, Cor. 5.2], $\sigma\left(T_{x}\right)=\sigma\left(T_{y}\right)$.

Now we prove the main result.

Theorem 3. Assume $T \in \mathscr{B}_{p, s}$. Then $r\left(T_{x}\right)$ is a continuous function on $(p, s)$.

Proof. If $r\left(T_{y}\right)=0$ for some $y \in(p, s)$, then $r\left(T_{x}\right)=0$ for all $x \in(p, s)$ by Proposition 1. Thus, we may assume $r\left(T_{x}\right)>0$ for all $x \in(p, s)$. For each $n \geq 1$, define

$$
\varphi_{n}(w)=n^{-1} \log \left(\left\|\left(T^{n}\right)_{w^{-1}}\right\|\right) .
$$

By the Riesz Convexity Theorem [6, Theorem 11, p. 525] $\varphi_{n}$ is a convex function on $\left(s^{-1}, p^{-1}\right)$. Now $\varphi_{n}(w)$ converges pointwise to $\varphi(w)=\log \left(r\left(T_{w^{-1}}\right)\right)$. By hypothesis $\varphi(w)$ has only finite values, and $\varphi$ must be convex on $\left(s^{-1}, p^{-1}\right)$. It follows from [10, Theorem 3.2] that $\varphi(w)$ is continuous on $\left(s^{-1}, p^{-1}\right)$, and thus, $r\left(T_{x}\right)$ is continuous on $(p, s)$.

Concerning this result, note that Example I shows that $x \rightarrow r\left(T_{x}\right)$ need not be continuous on the closed interval $[p, s]$.

Next we derive some consequences of the continuity of the spectral radius function. The first is a type of upper semicontinuity property for the function $x \rightarrow \sigma\left(T_{x}\right)$. For $K$ a compact subset of $\mathbf{C}$, let $\hat{K}$ denote the polynomial convex hull of $K$. E. Stout's book [11] is a good source for information on polynomial convexity.

Theorem 4. Assume $T \in \mathscr{B}=\mathscr{B}_{p, s}$ and fix $x \in(p, s)$. Let $U$ be an open set in C with $\sigma\left(T_{x}\right)^{\wedge} \subseteq U$. Then $\exists \delta>0$ such that $\sigma\left(T_{y}\right) \subseteq U$ whenever $|y-x|<\delta$.

Proof. Suppose no such $\delta$ exists. Then we can choose a sequence $\left\{y_{n}\right\} \subseteq$ $(p, s)$ with $y_{n} \rightarrow x$ such that for each $n$ there exists $\lambda_{n} \in \sigma\left(T_{y_{n}}\right)$ with $\lambda_{n} \notin$ $U$. The sequence $\left\{\lambda_{n}\right\}$ is contained in the compact set $\sigma_{\mathscr{B}}(T)$, and so some subsequence converges to a number $\lambda \notin U$. We may assume that $\lambda_{n} \rightarrow \lambda$.

Set $K=\sigma\left(T_{x}\right)^{\wedge}$, and if $q$ is a polynomial, then let $\|q\|_{K}=\sup \{|q(\mu)|: \mu \in$ $K\}$. Now $\lambda \notin K$, so by definition there is a polynomial $q$ such that $|q(\lambda)|>$ $\|q\|_{K}=1$. Choose a positive integer $n$ sufficiently large that $\left|q^{n}(\lambda)\right|>2$. Set $p=q^{n}$. Thus,

$$
|p(\lambda)|>2 \text { and }\|p\|_{K}=1 .
$$


Since $r\left(p\left(T_{x}\right)\right)=1$, by the continuity of the spectral radius (Theorem 3 ) $r\left(p\left(T_{y_{k}}\right)\right) \rightarrow 1$. Also, $p\left(\lambda_{k}\right) \in \sigma\left(p\left(T_{y_{k}}\right)\right)$ and $p\left(\lambda_{k}\right) \rightarrow p(\lambda)$. This provides a contradiction since for $k$ sufficiently large

$$
r\left(p\left(T_{y_{k}}\right)\right)<2 \text { and }\left|p\left(\lambda_{k}\right)\right|>2 .
$$

Next we prove a stronger upper semicontinuity property of the map $x \rightarrow$ $\sigma\left(T_{x}\right)$ which holds under the assumption that the spectrum of $T$ in the algebra $\mathscr{B}_{p, s}$ is "thin". First we need a preliminary result that uses the continuity of the spectral radius function.

Lemma 5. Assume $T \in \mathscr{B}=\mathscr{B}_{p, s}$ and $\lambda \notin \sigma_{\mathscr{B}}(T)$. Then $d(x)=\operatorname{dist}\left(\lambda ; \sigma\left(T_{x}\right)\right)$ is a continuous function on $(p, s)$.

Proof. For all $x \in(p, s)\left((\lambda-T)^{-1}\right)_{x}=\left(\lambda-T_{x}\right)^{-1}$. Thus since

$$
\sigma\left(\left(\lambda-T_{x}\right)^{-1}\right)=\left\{(\lambda-\mu)^{-1}: \mu \in \sigma\left(T_{x}\right)\right\},
$$

it follows that for $x \in(p, s)$

$$
r\left(\left((\lambda-T)^{-1}\right)_{x}\right)=\sup \left\{|\lambda-\mu|^{-1}: \mu \in \sigma\left(T_{x}\right)\right\}=(d(x))^{-1} .
$$

By Theorem 3 this function is continuous on $(p, s)$ which proves the lemma.

Theorem 6. Assume $T \in \mathscr{B}=\mathscr{B}_{p, s}$ and that $\sigma_{\mathscr{B}}(T)$ has empty interior. If $x \in(p, s)$ and $U$ is an open set with $\sigma\left(T_{x}\right) \subseteq U$, then $\exists \delta>0$ such that $\sigma\left(T_{y}\right) \subseteq U$ whenever $|x-y|<\delta$.

Proof. Suppose no such $\delta$ exists. Then there is a sequence $\left\{y_{n}\right\} \subseteq(p, s)$ such that $y_{n} \rightarrow x$ and for each $n$ there exists $\lambda_{n} \in \sigma\left(T_{y_{n}}\right)$ with $\lambda_{n} \notin U$. We may assume (by taking a subsequence if necessary) that $\left\{\lambda_{n}\right\}$ converges to a number $\mu \notin U$. Let $\varepsilon=\operatorname{dist}\left(\mu ; \sigma\left(T_{x}\right)\right)>0$. Set $D=\{\alpha \in \mathbf{C}:|\alpha-\mu|<\varepsilon / 4\}$. Since $\sigma_{\mathscr{B}}(T)$ has no interior, we can choose $\lambda \in D$ with $\lambda \notin \sigma_{\mathscr{B}}(T)$. By Lemma $5 d(y)=\operatorname{dist}\left(\lambda ; \sigma\left(T_{y}\right)\right)$ is a continuous function on $(p, s)$. By the choices of $\varepsilon$ and $\lambda, d(x)>\varepsilon / 2$. But since $\lambda_{n} \rightarrow \mu$, we have $d\left(y_{n}\right)<\varepsilon / 2$ for all $n$ sufficiently large, a contradiction.

Next we turn to some results concerning the situation where $\sigma\left(T_{x}\right)^{\wedge}$ is disconnected or has an isolated point at some $x \in(p, s)$. What we prove is that this property extends to all $y$ in some neighborhood of $x$.

We need the following technical lemma.

Lemma 7. Let $K$ and $J$ be compact subsets of $\mathbf{C}$ with $\hat{K}$ and $\hat{J}$ disjoint. Then there exists $U$ and $V$, open sets with compact closure, such that

(i) $\hat{K} \subseteq U$ and $\hat{J} \subseteq V$; and

(ii) $\bar{U}$ and $\bar{V}^{\wedge}$ are disjoint.

Proof. For each $\mu \in \hat{J}$, choose a polynomial $q_{\mu}$ such that $\left\|q_{\mu}\right\|_{K}=1$ and $\left|q_{\mu}(\mu)\right|>3$. Let

$$
\begin{aligned}
V_{\mu} & =\left\{\lambda \in \mathbf{C}: \mid q_{\mu}(\lambda)>3\right\}, \quad \text { and } \\
U_{\mu} & =\left\{\lambda \in \mathbf{C}:\left|q_{\mu}(\lambda)\right|<2\right\} .
\end{aligned}
$$


Let $\left\{V_{u_{1}}, \ldots, V_{\mu_{n}}\right\}$ be a finite cover for $\hat{J}$. For convenience, in the notation that follows the subscript $\mu_{k}$ will be replaced by $k$. Note that $\hat{J} \subseteq \bigcup_{k=1}^{n} V_{k}$ and $\hat{K} \subseteq \bigcap_{k=1}^{n} U_{k}$. Choose $U$ with compact closure such that

$$
\hat{K} \subseteq U \subseteq \bar{U} \subseteq \bigcap_{k=1}^{n} U_{k}
$$

If $\lambda \in \overline{U^{\wedge}}$, then $\left\|q_{k}\right\|_{\bar{U}}<2$, so $\left|q_{k}(\lambda)\right|<2$ for $1 \leq k \leq n$. Thus, $\overline{U^{\wedge}} \subseteq$ $\bigcap_{k=1}^{n} U_{k}$. Since $\bigcap_{k=1}^{n} U_{k}$ and $\bigcup_{k=1}^{n} V_{k}$ are disjoint, it follows that $\bar{U}^{\wedge}$ and $\hat{J}$ are disjoint.

Now repeat the argument above with $\bar{U}$ in place of $J$ and $J$ in place of $K$. The argument proves $\exists V$ an open set with compact closure such that $\hat{J} \subseteq V$ and $\bar{V}^{\wedge}$ and $\bar{U}^{\wedge}$ are disjoint.

Theorem 8. Let $T \in \mathscr{B}_{p, s}$. Assume for some $x \in(p, s), \sigma\left(T_{x}\right)^{\wedge}$ is disconnected. Then $\sigma\left(T_{y}\right)$ is disconnected for all $y$ in some neighborhood of $x$.

Proof. Assume $K$ and $J$ are disjoint nonempty compact sets with $\sigma\left(T_{x}\right)^{\wedge}=$ $K \cup J$. Then $\hat{K}=K$ and $\hat{J}=J$, so by Lemma 7, there exist open sets $U$ and $V$ with compact closure such that $K \subseteq U, J \subseteq V$, and $\bar{U}^{\wedge}$ and $\bar{V}^{\wedge}$ are disjoint. By Theorem $4 \exists \delta>0$ such that $\sigma\left(T_{y}\right) \subseteq U \cup V$ whenever $|x-y|<\delta$. Fix $y$ with this property, and assume (without loss of generality) that $x<y$. Then $T \in \mathscr{B}=\mathscr{B}_{x, y}$. By [2, Theorem 5.1(4)]

$$
\sigma_{\mathscr{B}}(T) \subseteq \sigma_{\mathscr{B}}(T)^{\wedge}=\left(\sigma\left(T_{x}\right) \bigcup \sigma\left(T_{y}\right)\right)^{\wedge} \subseteq(\bar{U} \bigcup \bar{V})^{\wedge}=\bar{U} \bigcup \bar{V},
$$

where the last equality follows from [11, Lemma 29.21(b)]. Now since $\sigma\left(T_{x}\right)$ has nonempty intersection with both $U$ and $V$, the same is true for $\sigma_{\mathscr{B}}(T)$. Then using the fact that $S \rightarrow S_{y}$ is a continuous algebra monomorphism of $\mathscr{B}$ into $\mathscr{B}\left(L^{y}\right)$, it follows from [2, Theorem 4.5] that $\sigma\left(T_{y}\right) \cap U$ and $\sigma\left(T_{y}\right) \cap V$ are nonempty. Thus, $\sigma\left(T_{y}\right)$ is disconnected.

Corollary 9. Let $T \in \mathscr{B}_{p, s}$. Assume for some $x \in(p, s)$ that $\lambda_{0}$ is an isolated point of $\sigma\left(T_{x}\right)^{-}$. Then $\lambda_{0}$ is an isolated point of $\sigma\left(T_{y}\right)$ for all $y$ in some neighborhood of $x$.

Proof. Set $K=\left\{\lambda_{0}\right\}$ and $J=\sigma\left(T_{x}\right) \backslash\left\{\lambda_{0}\right\}$. Choose $U, V, \delta>0, y$, and $\mathscr{B}$ is in the proof of Theorem 8. Then $\sigma_{\mathscr{B}}(T) \subseteq \bar{U} \cup \bar{V}$. Let $Q \in \mathscr{B}$ be the spectral projection corresponding to the set $\sigma_{\mathscr{B}}(T) \cap \bar{U}$ which is a nonempty open and closed subset of $\sigma_{\mathscr{B}}(T)$. Then by the Spectral Mapping Theorem

$$
\sigma\left((Q T)_{x}\right)=\sigma\left(Q_{x} T_{x}\right)=\left\{\lambda_{0}\right\} .
$$

It follows from Corollary 2 that for all $w \in(x, y), \sigma\left((Q T)_{w}\right)=\left\{\lambda_{0}\right\}$. Therefore $\lambda_{0}$ is an isolated point of $\sigma\left(T_{w}\right)$ when $x<w<y$. This argument proves that $\lambda_{0}$ is an isolated point of $\sigma\left(T_{w}\right)$ whenever $|x-w|<\delta$.

Added in proof. It has been pointed out to me that a general upper semicontinuity property of the map $y \rightarrow \sigma\left(T_{y}\right)$ has been proved by I. Ya. Sneiberg in 
[Mat. Issled., 9 (1974), 214-229 (in Russian)]. Both Theorems 4 and 6 are special cases of this result. On the positive side, this same result implies that Theorem 8 and Corollary 9 have more general forms. Specifically, $\sigma\left(T_{x}\right)^{\wedge}$ can be replaced by $\sigma\left(T_{x}\right)$ in the hypotheses of these two results without altering the conclusions.

\section{REFERENCES}

1. B. Barnes, The spectrum of integral operators on Lebesgue spaces, J. of Operator Th. 18 (1987), $115-132$.

2. _ Interpolation of spectrum of bounded operators on Lebesgue spaces, to appear in Rocky Mt. J. Math.

3. __ Essential spectrum in a Banach algebra applied to linear operators, recently submitted.

4. D. Boyd, The spectrum of the Cesàro operator, Acta. Sci. Math. 29 (1968), 31-34.

5. K. Dayanithy, Interpolation of spectral operators, Math. Z. 159 (1978), 1-2.

6. N. Dunford and J. Schwartz, Linear operators, Part I, Interscience, New York-London, 1964.

7. D. Herrero, Operator theory, Advances and Applications, Vol. 11, Birkhauser-Verlag, Basel, 1983, 191-232.

8. J. Newburgh, The variation of spectra, Duke Math. J. 18 (1951), 165-176.

9. J. Nieto, On the essential spectrum of symmetrizable operators, Math. Annalen 178 (1968), 145-153.

10. W. Rudin, Real and complex analysis, Third Ed., McGraw-Hill, New York, 1987.

11. E. Stout, The theory of uniform algebras, Bodgen \& Quigley, Tarrytown-on-Hudson, New York, 1971.

Mathematics Department, University of Oregon, Eugene, Oregon 97403 